\title{
Specific Virology: Viruses as Diseases
}

\author{
Elisabeth Presterl, Magda Diab-El Schahawi, \\ Luigi Segagni Lusignani, Helga Paula, and Jacqui S. Reilly
}

\subsection{Historical Overview of the Development of Virology}

1796: Jenner transfers cowpox material from a milkmaid to a boy and shows evidence of immunity generated by re-vaccination with virulent pox material.

1885: With the development of a rabies vaccine and the implementation of the first vaccination in a person at risk, Pasteur founds rabies research.

1892: Ivanovsky notes the transferability of tobacco mosaic disease by the bacteria-free filtrate from pressed juices of infested leaves.

1956: Crick and Watson come to the conclusion by evaluating the X-ray structure of analytical studies and by theoretical considerations that in smaller viruses the nucleic acid is surrounded by regularly arranged protein subunits. This starts the research into the fine structure of viruses.

\section{Discovery of New Viruses}

- 1965: Hepatitis B-virus

- 1981: Parvovirus B19

- 1983: HIV-I, HHV-6/7

- 1988: Hepatitis C-virus

\footnotetext{
E. Presterl $(\bowtie) \cdot$ M. Diab-El Schahawi $(\bowtie) \cdot$ L. S. Lusignani $\cdot$ H. Paula Department of Hygiene and Infection Control, Medical University of Vienna, Vienna, Austria e-mail: elisabeth.presterl@meduniwien.ac.at; magda.diab-elschahawi@meduniwien.ac.at; luigi.segagnilusignani@meduniwien.ac.at; helga.paula@meduniwien.ac.at

J. S. Reilly

Glasgow Caledonian University, Glasgow, UK

e-mail: jacquelinereilly@nhs.net
} 
- 1993: Herpes virus Typ 8

- 2003: SARS-coronavirus

- 2012: MERS-coronavirus

\subsection{Adenoviruses}

- No enveloped DNA virus

- 51 human-pathogenic serotypes

- High environmental resistance

Path of infection: transmission by droplets or smear infection in the water or instruments.

Incubation period: varies from a few days to 10 days.

Clinical manifestation: Adenoviruses can infect the epithelial cells of the oropharynx, respiratory, gastrointestinal tract and eye. The infection is often asymptomatic or with mild symptoms.

The various human-pathogenic serotypes cause numerous diseases which mainly affect the respiratory tract ("influenza infection"), the eye (conjunctivitis) or the gastrointestinal tract (diarrhoea). Respiratory tract infections: a noteworthy part of all "colds" of infants is caused by adenoviruses. Young adults can develop from respiratory infections to life-threatening pneumonia. School-age children may experience febrile pharyngitis and conjunctivitis as part of pharyngoconjunctival fever.

Eye infections: Acute follicular conjunctivitis (so-called swimming pool conjunctivitis), there is a one-sided, often painful reddening of the eye on both sides and periauricular swelling of the lymph nodes. Inadequate quality of the pool water or inadequate sterilization or disinfection of instruments during ophthalmological activities may lead to transmission of adenoviruses.

Intestinal infections: Pronounced diarrhoea. In infants, adenoviruses are the second leading cause of diarrhoea after rotaviruses.

Other infections: Infections of the urogenital tract (acute haemorrhagic cystitis), less commonly intestinal invaginations and meningoencephalitis.

\subsection{Parvoviruses: Parvovirus B19}

No enveloped DNA virus

Path of transmission: Infected respiratory droplets.

Pathogenesis and clinical presentation: The virus damages the erythroblasts, and there is a drop in reticulocytes along with transient anaemia. In childhood, erythema infectiosum generally referred to as the "fifth disease", pertaining to the five major exanthematous childhood diseases: scarlet fever, measles, rubella and exanthema subitum. Parvovirus is characterized by a rash which begins classically on the face ("slapped cheek syndrome") and spreads on the extensor sides of the extremities in a ring-shaped pattern. 
Delayed complications: Arthritis and arthralgia.

Parvovirus B19 in pregnancy: Can cause intrauterine infection in about $20 \%$ of cases. In early pregnancy, this can lead to spontaneous abortion of the foetus. In the second and third trimester, there is a hydrops fetalis, which, if left untreated, usually leads to the death of the foetus. Hydrops fetalis characteristically presents with pronounced cerebral oedema and soft tissue oedema, ascites and maceration of the foetus due to the severe oxygen deficiency of the tissue due to virus-induced anaemia.

Prophylaxis and treatment: Exposure prophylaxis for pregnant women (it should be noted that the exanthematous stage rarely poses a risk of infection). There is no specific antiviral therapy. In the case of exposure to the virus during pregnancy, maternal serostatus (immunity) should be ascertained. In the case of suspected transplacental infection, detection of the virus in maternal blood/serum as well as in umbilical cord blood, amniotic fluid or in chorion biopsy material should be considered.

\subsection{Enteroviruses}

- No enveloped RNA viruses

- Part of the enterovirus group:

- Poliovirus type 1-3

- Coxsackie virus A and B

- Echovirus

- Human enterovirus

- High environmental resistance

- Transmission faecally-orally

- Massive viral shedding through asymptomatic but infectious persons

Increased incidence in the summer months; also referred to as "summer flu". Most enterovirus infections are asymptomatic or with the nonspecific clinical presentation of a flu infection.

\section{Typical Clinical Manifestations of Enterovirus Infections}

Pathogenesis: Enteroviruses multiply after ingestion first in the local tissue of the nasopharynx and later in the intestine. The viruses reach the target organs either via the bloodstream (viremia) or via neural spread. However, only in a very small percentage does organ manifestation occur with the above clinical signs (see Table 13.1).

\section{Clinical Presentation}

Poliovirus: The spinal form of poliomyelitis causes irreversible flaccid paralysis of the limb musculature, the chest muscles or the diaphragm, and thus poses a risk for suffocation. The bulbar form of poliomyelitis causes disruption of the respiratory and circulatory centre. There is also an encephalitic form which, like the bulbar form, can quickly lead to death. Infections in pregnant women acquired shortly before birth can be transmitted to the child and lead to sepsis-like illnesses of the new-born. 
Table 13.1 Clinical manifestations of enterovirus infections

\begin{tabular}{l|l}
\hline Viruses & Clinical manifestations \\
\hline All enteroviruses & Fever, head and joint aches ("summer flu“) \\
\hline Poliovirus & Paralysis, myelitis, meningitis, encephalitis \\
\hline $\begin{array}{l}\text { Coxsackie-A- } \\
\text { viruses }^{\mathrm{a}}\end{array}$ & $\begin{array}{l}\text { Respiratory diseases, pneumonia (infants), herpangina (toddlers), hand-foot- } \\
\text { mouth illness, gingivostomatitis, myocarditis, haemorrhagic conjunctivitis, } \\
\text { exanthema }\end{array}$ \\
\hline $\begin{array}{l}\text { Coxsackie-B- } \\
\text { viruses }^{\mathrm{a}}\end{array}$ & Diarrhoea, myocarditis, pericarditis \\
\hline ECHO-viruses $^{\mathrm{a}}$ & Maculopapular rash, myocarditis, gastroenteritis \\
\hline $\begin{array}{l}\text { Other } \\
\text { enteroviruses }^{\mathrm{a}}\end{array}$ & $\begin{array}{l}\text { Bronchiolitis, pneumonia, hand-foot-mouth illness, haemorrhagic } \\
\text { conjunctivitis }\end{array}$ \\
\hline
\end{tabular}

${ }^{\mathrm{a}}$ All enteroviruses have the ability to cause polio-like symptoms (meningitis, meningoencephalitis, paralysis)

Coxsackie-A-viruses: Causes herpangina especially in infants, which manifests itself by the formation of painful small blisters on the oral mucosa, high fever and loss of appetite. The hand-foot-mouth disease, which is also caused by Coxsackie A viruses, causes blisters on the hands, feet and in the mouth.

Coxsackie-B-viruses: Cause myositis of the abdominal and chest muscles with a dry pleurisy (breathing extremely painful).

Enteroviruses: Often cause of aseptic (abacterial) meningitis, and often cause more or less discrete rashes.

Prophylaxis: Prophylaxis of poliomyelitis through active immunization.

Parenteral vaccine by inactivated polio viruses according to Salk, which contains inactivated polioviruses of all three serotypes. Primary immunization should be done in infancy. Booster vaccinations should be in accordance with the Austrian vaccination plan. Sabin's oral polio vaccine, which is no longer available in Austria, contains attenuated, replicable viruses of all three types.

All other enteroviruses: Prophylaxis consists of hygienic measures.

\subsection{Hepatitis A Virus}

- No enveloped RNA virus

- Transmission: Faecally-orally

This virus is extremely stable against heat and other environmental factors. The virus can enter the food chain through fertilization of crops with faecal matter or through contaminated wastewater, which can lead to an accumulation in mussels and oysters and play a role in outbreaks. Hepatitis A virus is endemic in countries with poor hygienic conditions. 
Incubation period: 2-6 weeks.

Pathogenesis and clinical presentation: The hepatitis A virus enters the gastrointestinal tract through smear infection or through contaminated food/drinking water and reaches the liver via the bloodstream.

In children: Over $90 \%$ of cases manifest subclinically.

In adulthood: In 70-80\% of cases acute hepatitis occurs (loss of appetite, fatigue, fever, vomiting, diarrhoea, bilirubinuria and jaundice).

There are no chronic courses. There is low lethality (in children about $1 \%$, in adults about $5 \%$ ).

Diagnosis: Hepatitis A antibody detection in serum, detection of virus antigen from stool, detection of the viral RNA genome by PCR in serum or stool.

Prophylaxis: Active vaccination with an attenuated vaccine (2 doses in separate intervals of at least 4 weeks) provides long-term protection. Vaccination is recommended for people working in health care, day care, kitchens as well as sewerage and sewage treatment workers and travelers in endemic areas.

\subsection{Human Rhinovirus}

- No enveloped RNA virus

- Transmission: Through droplet or smear infection directly from person to person

- Incubation period: 1-4 days

- Over 100 serotypes

- Propagation in the mucous membranes of the nasopharynx

Clinical presentation: Runny nose with sneezing, coughing and watery to mucous secretion discharge. Disease duration is usually 1 week.

\subsection{Rotavirus}

- No enveloped RNA virus

- High environmental resistance

- Transmission: Faecally-orally

- Most common pathogen of diarrhoea in children!

Clinical presentation: After an incubation period of 2-3 days, patients present with watery diarrhoea, vomiting and fever for about 4-7 days. Humans excrete the viruses over a period of up to 14 days!

Diagnosis: Antigen detection in stool.

Prophylaxis: Oral vaccination from the sixth week of age, depending on the vaccine will be given 2 or 3 doses with a minimum interval of 4 weeks. 


\subsection{Herpes Viruses}

- Herpes simplex virus $1 \& 2$

- Varicella-zoster virus (VZV)

- Human cytomegalo virus (CMV)

- Epstein-Barr virus (EBV)

- Human herpes virus 6-8

\section{General}

- Enveloped DNA virus

- Herpes viruses are widespread in humans and animals

- Persist lifelong in the infected host

- After initial infection, the virus genome remains in the body for life (latent infection)

- Latency is periodically interrupted by reactivation and viral regeneration

- The frequency and duration of virus reactivation depends on the type of herpes virus, the latent site and the immune status

\subsubsection{Herpes Simplex Virus Type 1 (Herpes Labialis)}

Primary infection usually occurs in infancy and is predominantly asymptomatic; it rarely manifests as stomatitis aphthosa (= oral blight).

Acute herpetic gingivostomatitis: painful clinical picture with fever, lymphadenopathy and ulcerative lesions of the oral mucosa, tongue and lips, occurring in about $1 \%$ of primary infections.

In the course of the asymptomatic or symptomatic primary infection, neuronal spread occurs via the axons and latent infection of the neurons of the trigeminal nerve. During the later reactivation, infectious virions reappear in the area of the nerve endings, resulting in cold sores near the original site of infection (herpes labialis). The blister content is an important source of new infections.

\subsubsection{Herpes Simplex Virus Type 2 (Herpes Genitalis)}

Path of infection: The transmission takes place through sexual contacts; the primary infection usually takes place in adolescence or adulthood.

Clinical manifestation: Blistering and small ulcers occur in the male genital area and in the area of the vulva and vagina in women, occasionally also perianal and rectally. In herpes simplex virus-type 2 lifelong latent in the lumbosacral ganglia and upon reactivation presents as genital herpes.

Complications: Herpes simplex virus encephalitis can occur both at primary infection and at reactivation. 
- Keratoconjunctivitis herpetica: Primarily an inflammation of the cornea of the eye (keratitis) and possibly also the conjunctiva (keratoconjunctivitis) by herpes simplex infection. Herpes simplex virus 1 (HSV-1) is predominantly the causative agent, rarely HSV-2. Recurrence can lead to irreversible scarring.

- Eczema herpeticatum: In atopic dermatitis or chronic eczema, pre-damaged (and thus particularly virus-sensitive) skin can present with extensive blistering.

- Herpes neonatorum: It is especially problematic when a woman's first contact with herpes simplex virus type 2 occurs during pregnancy, since it increases the risk of a perinatal infection of the new-born child with herpes neonatorum. Since there are no maternal antibodies yet in a primary infection, the virus can spread particularly well in the immature new-born. Infections of the new-born can range from local infections of the skin and mucous membranes to generalized infection involving the internal organs and the CNS. Most of the therapy comes too late to prevent a fatal outcome. If genital herpes is suspected, delivery by caesarean section is indicated.

However, if the pregnant woman experiences recurrence of genital herpes at the time of birth, the child may become infected in the birth canal, but the maternal antibodies transferred by the placenta prevent subsequent viremia. Nevertheless, neonatal encephalitis with a high lethality may occur.

\section{Varicella Zoster Virus}

Path of infection: The virus is transmitted aerogenically via droplets of blister fluid and leads to a lifelong persistence. Since the virus is highly contagious, already a short-term stay in a room in which a varicella patient is exposed is sufficient for disease transmission. Contagiousness begins 1-2 days before the onset of the rash and ends 5-7 days after the onset of last rashes. Herpes zoster sufferers are contagious until the encrustation of the blisters (smear infection).

\section{Clinical Symptoms}

The varicella zoster virus causes two different clinical pictures:

Varicella (chicken pox) during initial infection and herpes zoster (shingles) in a reactivation.

Varicella: After an uncharacteristic prodromal stage (1-2 days before onset of illness), the disease begins with itchy rash and fever for a period of 3-5 days. The skin lesions consist of poplars, vesicles and scabs at various stages of development ("starry sky"). The lesions may quickly develop into blisters that appear first on the trunk and face and can spread rapidly to other parts of the body, including the mucous membranes and hairy scalp. Varicella usually has a benign course in healthy individuals and usually heals without scarring. However, severe scratching or bacterial superinfections can cause scarring. New-borns and immunocompromised individuals may experience severe haemorrhagic disease progression, often with a fatal outcome. 


\section{Complications}

- Bacterial superinfection mostly caused by Streptococcus pyogenes or Staphylococcus aureus

- Varicella pneumonia

- CNS manifestation

In isolated cases myocarditis, corneal lesions, nephritis, arthritis, bleeding tendencies, acute glomerulonephritis and hepatitis.

\section{Foetal Varicella Syndrome}

If varicella occurs in the first and second trimesters of pregnancy, there may be a foetal varicella syndrome characterized by segmental skin lesions (ulcers, scars), neurological disorders and malformations (atrophy of the brain, paresis, seizures), eye damage and skeletal abnormalities.

Severe neonatal chickenpox: May result from a primary infection of the susceptible mother within 5 days of birth or up to $48 \mathrm{~h}$ thereafter. Since the new-born has an immature immune system and in these cases no protective is among new-borns who contract varicella between the fifth and 12th day of life.

\section{Herpes Zoster}

Herpes zoster is not an exogenous new infection, but an endogenous recurrence. The virus can only reactivate in people with a prior varicella zoster virus infection. The virus persists in the spinal or cranial nerve ganglia and can eventually lead to a reactivation in the form of herpes zoster. It occurs predominantly in immunocompromised or elderly persons. Herpes zoster is characterized by a unilateral vesicular eruption that is limited to a dermatome with sometimes very severe pain. Infestation of the trigeminal nerve (ophthalmic nerve) leads to the zoster ophthalmicus. Furthermore it can exhibit in the form of zoster oticus, zoster maxillaris or zoster genitalis.

Preventive measures: A varicella vaccine is available from the age of 2 , two vaccinations are given at intervals of at least 4 weeks. It is also recommended to vaccinate unvaccinated 9-17-year-olds without varicella history, as the disease is associated with a higher complication rate.

Post-exposure prophylaxis by passive immunization with varicella zoster immunoglobulin is recommended within $96 \mathrm{~h}$ of exposure (face to face contact or household contact) for persons at increased risk of varicella complications. This group includes unvaccinated pregnant women without varicella history, immunodeficient patients with unknown or missing varicella immunity, new-borns whose mothers have varicella 5 days before to 2 days after confinement.

\section{Remember}

- Primary infection of the mother with varicella zoster virus: Viremia $\rightarrow$ dangerous for the child!

- Endogenous reactivation (herpes zoster): No viremia $\rightarrow$ safe for the child! 


\subsubsection{Cytomegalovirus (CMV)}

CMV exclusively infects human cells and proliferates slowly.

After primary infection, the CMV virus persists for life in latent form in various cells and organs and often reactivates. Both primary infection and reactivation are usually asymptomatic. However, it can lead to severe disease courses under immunosuppression. CMV infection remains one of the most important prenatal viral infections (congenital disease).

\section{Infection}

Since the virus may be present in tear fluid, saliva, urine, genital secretions as well as breast milk and blood, possible transmission upon contact with infectious bodily fluids, e.g., by breastfeeding, kissing, sexual contacts, but also by blood products and organ transplants is possible. During lactation, CMV is excreted by almost all seropositive women with milk and passes to the children at a rate of about $35 \%$.

Mostly, oral transmission is by saliva or other bodily fluids (urine, breast milk, cervical secretions, seminal fluid). Sexual contacts are another important source of infection. Other important transmission pathways are the prenatal or perinatal infection from the mother to the child.

\section{Clinical Manifestation and Pathogenesis}

In immunocompetent individuals, CMV infection is usually asymptomatic or has nonspecific symptoms (such as flu-like respiratory symptoms, fatigue, fever, cough). Even women who become infected with CMV during pregnancy have the majority (about $75 \%$ ) no symptoms.

Complications may occur especially in new-borns or those with congenital or acquired immunodeficiency, as well as immunosuppressive therapy, in which the infection can damage many organ systems. These include in particular the lungs with the risk of developing pneumonia, the liver, the intestine and the eye, which can lead to retinal infection (retinitis) with blindness.

In new-borns that have been infected in utero, growth delays and especially hearing damage may occur, also often late neurological damage is observed.

Prenatal infection: In the first infection of pregnant women with high viremia, the virus is transmitted diaplacentally in about $40-50 \%$ of cases. In about $5-10 \%$ of cases there is a clinical manifestation.

\section{For Clinical Manifestations}

Cerebral damage (microcephaly, hearing loss, optic atrophy, seizures, spasticity or motor disorders), hepatosplenomegaly with jaundice, haemorrhage, haemolytic, anaemia Interstitial pneumonia, in the worst case intrauterine foetal death.

Perinatal infection: Infection occurs either at birth or through breast milk. The perinatal infection of the new-born usually proceeds subclinically with occasional long-term consequences such as hearing disorders or behavioural problems. Premature babies are very at risk and must be protected by exposure prophylaxis. 
Postnatal infection: It is usually subclinical in children and often mild in adults. In most cases, a long-term fever (status febrilis) occasionally shows a similar mononucleosis with involvement of the liver.

Complications occur mainly in immunocompromised individuals (pneumonia, retinitis, nephritis, encephalitis) and in prenatal infections.

\subsubsection{Epstein-Barr Virus (EBV)}

Path of infection: Transmission is mainly via saliva.

EBV affects the lymphocytes which are stimulated for proliferation. The EBV primary infection therefore leads to lympho-proliferation which cannot be controlled by immunosuppression or genetic immune deficiencies. Burkitt's lymphoma and part of Hodgkin's lymphoma are EBV-associated B-cell lymphomas. Worldwide, about $90-95 \%$ of the adult population is infected.

Clinic and pathogenesis: As the virus is transmitted mainly in the saliva, the primary infection usually occurs in early childhood and is usually asymptomatic. In older children and young adults, it often comes to the clinical picture of infectious mononucleosis (Pfeiffer's glandular fever, kissing disease). Infectious mononucleosis is characterized by fever, swelling of the lymph nodes, hepatosplenomegaly, white matter tonsillitis, and in some cases maculopapular rash of the skin occurs, especially after the administration of certain antibiotics.

\subsubsection{Human Herpes Viruses 6, 7 Und 8}

\subsubsection{Human Herpes Virus 6}

The virus is widespread and transmitted by saliva in early infancy. The prevalence rate for the 3-year-olds is already up to $95 \%$. HHV-6 is the causative agent of the exanthema subitum (roseola infantum, 3-day fever).

Clinic: Primary infection in infancy usually remains asymptomatic. In infancy, exanthema subitum (3-day fever) may occur after an incubation period of 5-15 days. Classically, there is an abrupt increase in fever to $40-41{ }^{\circ} \mathrm{C}$ with febrile convulsions and a rapid defilement after 3 days. Afterwards a fleeting rash appears.

Complications: Rarely, meningitis, encephalitis, hepatitis severe events occur especially in immunosuppression in organ or bone transplant patients.

\subsubsection{Human Herpes Virus 7}

Like the HHV-6, it is widespread and transmitted by saliva. Primary infection of infants occurs somewhat later than with HVV-6 and may occasionally go below the exanthema subitum picture. Both initial infection and reactivation in immunosuppression are usually asymptomatic.

\subsubsection{Human Herpes Virus 8}

Transmission occurs probably through saliva and sexually. Participation in the development of Kaposi's sarcoma and other lymphomas in serous body cavities (pleura, pericardium, peritoneal cavity) is considered a definitive diagnosis. 


\subsection{Influenza Viruses}

These are enveloped RNA viruses. Pathogens of influenza are orthomyxoviruses which are subdivided into types $\mathrm{A}, \mathrm{B}$ and $\mathrm{C}$. Especially relevant to humans are influenza $\mathrm{A}$ and $\mathrm{B}$ viruses. Influenza $\mathrm{C}$ viruses are virtually meaningless and cause only mild childhood disease.

Influenza A and B viruses are characterized by spike-like surface structures formed by the surface glycoproteins, hemagglutinin (HA) and neuraminidase (NA). There are 16 different HA and 9 NA known. Influenza A viruses are named by type and subtype, e.g., A (H1N1). There are no subtypes of influenza B, but in recent years there are two genetically distinct lines circulating worldwide (Yamagata line and Victoria line).

Reservoir: Influenza A viruses occur in humans and in addition also in mammals (for example, pigs, horses). Influenza B viruses only occur in humans. The actual reservoir of influenza A viruses are birds, especially waterfowl, in which all previously known subtypes could be detected. A distinction is made between avian influenza viruses (which occur in birds) and human ones (which circulate in the human population) differ in that they bind very specifically to different receptors in the upper respiratory tract of humans and birds, whereas in the respiratory tract of the pig receptors for both human and avian influenza viruses.

\section{Mutations and Antigen Drift, Reassortment and Antigen Shift}

On the one hand, the great genetic variability of the influenza viruses is based on the fact that the eight gene segments are freely combinable and, on the other, on the high mutation rate. Point mutations lead to antigenic drift (antigenic shift). If the surface antigens HA and NA, which are important for the immune response, are affected by point mutation, the viruses are poorly or no longer recognized by the antibodies present. Since there is only a long-lasting immunity against viruses with a very high genetic relationship, the continuously occurring drift variants can cause annual flu outbreaks. Therefore, every year for all vaccine antigens must be checked whether they differ from the previous viruses and vaccinations must be adapted to the drift variant.

In contrast, antigen shift results in a complete replacement of an entire HA or NA genome segment by reassortment. The antigen shift leads to a complete re-equipment of the surface of the virus with hemagglutinin and/or new neuraminidase molecules, against which surface molecules there is no immunity in the population.

Occurrence: Influenza virus infections are common worldwide. In the temperate zones of the northern and southern hemisphere, influenza waves occur regularly in the respective winters. Due to the seasons set by 6 months, the winter influenza wave in the southern hemisphere occurs when summer is in the northern hemisphere and vice versa. It can also occur out of season flu outbreaks.

Transmission: Influenza viruses are transmitted by droplet infection (cough or sneezing) and smear infections (hands). The viruses multiply locally in the epithelial cells of the upper and lower respiratory tract lead to a direct damage to the infected cells. The destruction of the cells causes a strong inflammatory reaction as a result of which bacterial superinfections (for example, with Staphylococcus aureus, Streptococcus pneumoniae or Haemophilus influenzae) can occur. 


\section{Incubation Period: 1-2 Days}

Duration of contagiousness: On average about 4-5 days from the onset of the first symptoms. A longer duration is possible, e.g., in children, persons with chronic preexisting conditions. From a pragmatic point of view, individuals with influenza are recommended to be isolated for 7 days.

Clinical manifestation: The typical influenza symptoms are characterized by:

- Sudden onset of illness with fever $\left(\geq 38.5^{\circ} \mathrm{C}\right)$

- Dry, irritating cough

- Sore throat

- Muscle and/or headache

- Other symptoms may include: General weakness, sweats, nausea, vomiting and diarrhoea

It should be noted, however, that not all affected influenza patients experience the above symptoms. Roughly speaking, one-third of infections lead to a febrile manifestation, one-third to mild and the last third to asymptomatic clinical presentation.

\section{Complications}

Rarely, severe courses occur, with pulmonary complications (pneumonia!) In the foreground. If other organs are involved, myositis, rhabdomyolysis, encephalitis, myocarditis may occur. In children, complications of the CNS were also observed.

\section{Diagnosis}

Gold standard is the genome detection by means of PCR from nasal or throat swab, bronchial lavage. Furthermore, rapid tests for the direct virus antigen detection from nose/throat material are available. But with a lower specificity and sensitivity than the PCR virus isolation by culture is usually reserved for specialized laboratories. Further methods for the detection of viral antigens are the immunofluorescence and ELISA test which are comparable in their sensitivity with the fast text.

\section{Therapy}

Nowadays almost exclusively neuraminidase inhibitors are used therapeutically. They are active against both influenza A and influenza B viruses.

\section{Prophylaxis}

Vaccination-Recommended is the annual active immunization using the dead vaccine prior to the influenza season in the fall. Target group for vaccinations: persons over 60 years, pregnant women and women who want to become pregnant during the influenza season, children from the age of 6 months, all persons with increased risk as a result of chronic diseases (chronic lung, heart and circulatory diseases, kidney disease, neurological diseases, metabolic diseases and immune deficiencies), caregivers (e.g. in hospitals, nursing homes) and household contacts of risk groups, in particular persons from health professions. 


\section{Parainfluenza Virus}

Enveloped RNA virus.

Four serotypes - Type 1-4, Type 1-3 are distributed worldwide, Type 4 mainly in the Americas.

Transmission: By droplet infection and direct contact.

Incubation period: 3-5 days.

Clinic: The viruses lead to a strong inflammation and swelling of the mucous membranes and trigger flu-like symptoms. Feared are severe cases, which occur more frequently in infancy, resulting in acute laryngotracheobronchitis with inspiratory stridor, barking cough, hoarseness and dyspnoea (pseudo-krupp). In infants, the descending infection can lead to bronchiolitis and pneumonia. Serious diseases occur in highly immunosuppressed patients (bone marrow transplantation).

Diagnostics: Serological procedures (seroconversion or a significant title increase in paired sera), virus isolation or PCR or immunofluorescence.

Prophylaxis and therapy: Currently none available. Treatment is based on symptomatic measures.

\section{Respiratory-Syncytial Virus (RSV)}

The enveloped RNA virus belongs to the family Paramyxoviruses.

Transmission occurs through droplet infection as well as smear infection (contaminated objects).

Clinical manifestation: The RS virus causes respiratory infections in infants and toddlers, which occur seasonally-often in late autumn and winter. RSV is the main agent of bronchiolitis in infants in the first months of life. Since it is highly contagious, it is often the cause of healthcare-associated infections in children's hospitals and preterm stations. The likelihood of contact with the virus in the first months of life is already very high. There are also reinfections possible. Particularly at risk are infants in the first months of life, as well as older infants and toddlers with pre-existing conditions (congenital heart defects cystic fibrosis, bronchial asthma).

Serious infections also occur in older persons with pre-existing conditions as well as in immunosuppressed patients. These patients can often cause fatal pneumonia. Often the virus also leads to a middle ear infection which can be superinfected bacterially.

Diagnosis: The virus can be detected by virus culture, PCR or direct immunofluorescence.

\subsection{Mumps Virus}

The enveloped RNA virus belongs to the family Paramyxoviruses and occurs only in humans.

Transmission: Aerogen by droplet infection and direct salivary contact, rarely by saliva-contaminated objects.

Incubation period: 16-18 days. 
Duration of infectiousness: The infectious is greatest 2 days before to 4 days after onset of disease. Overall, an infected person may be contagious 7 days before to 9 days after the occurrence of parotid gland swelling. Clinically unapparent infections are also contagious.

Clinical manifestations: Mumps (Parotitis epidemica) is a systemic infectious disease and can occur at any age. A disease usually leads to lifelong immunity.

The mumps disease is typically characterized by a painful one-sided (20-30\%) or double-sided (70-80\%) inflammatory swelling of the parotid, lasting about 3-8 days. In up to $15 \%$ of cases, involvement of the submandibular or sublingual salivary glands is observed. The infection may be preceded by a multi-day prodromal stage with fever, headache, malaise, myalgia and loss of appetite. In children under 2 years of age, the majority of mumps infections are subclinical. In children under 5 years, the mumps infection often presents as an acute respiratory disease (up to $50 \%$ of cases). At least $30-40 \%$ of infections are clinically unapparent or subclinical.

In the context of the disease various complications can occur that become more common with age.

Complications include:

- CNS involvement (e.g. encephalitis, meningitis)

- Deafness

- Orchitis, which can lead to sterility in the adult male

- Mastitis

- Pancreatitis

- Nephritis

- Myocarditis

Diagnostics: Diagnosis is frequently done based on the typical clinical manifestation of parotic gland swelling. For a more reliable diagnosis serology and PCR are available.

Therapy: There is no specific antiviral therapy for mumps. The therapy is exclusively symptomatic.

Prophylaxis: A live vaccination (measles, mumps, rubella) is available. From the age of 10, all children should be vaccinated against mumps, measles and rubella with two vaccinations at least 4 weeks apart.

\section{Measles Virus}

The enveloped RNA virus belongs to the family Paramyxoviruses. The virus is extremely environmentally unstable (very sensitive to external influences such as elevated temperature, light and disinfectants). The measles virus is distributed worldwide, the natural reservoir are humans.

Pathway of infection: Measles is one of the most infectious diseases transmitted by inhalation of infectious droplets (talking, coughing, sneezing) - airborne transmission - as well as by contact with infectious secretions from the nose or throat. Already on short exposure the measles virus leads to an infection (contagion index 
near $100 \%$ ) and triggers clinical symptoms in more than $95 \%$ of the unprotected infected persons.

Incubation period: 8-10 days to the onset of the catarrhal stage, 14 days to the onset of the rash.

Duration of contagiousness: $3-5$ days prior to the onset of the rash until 4 days after the appearance of the rash. Infectiousness is greatest immediately before the appearance of the rash.

Clinical manifestation: Measles is a systemic viral infection with a biphasic course. It starts with fever, conjunctivitis, runny nose, cough and an enanthema on the oral mucosa. These so-called Koplik spots (lime-like white to blue-white spots) are typical of measles. Measles maculopapular exanthema (brownish-pink confluent patches) is between the third and seventh day after the onset of the initial symptoms. Classically, it starts in the face and behind the ears and persists for about 4-7 days. When decaying, a bran-like scaling is often observed. The temperature drop occurs between the fifth and seventh day of illness. A measles disease leaves a lifelong immunity.

Complications: Infection with measles causes a transitory immunodeficiency for about 6 weeks. As a consequence, there is a temporary increase in susceptibility to bacterial superinfections (measles-associated otitis media, bronchitis, pneumonia and diarrhoea are common). In about $0.1 \%$ of cases, there is dreaded post-infective encephalitis. With disturbance of consciousness up to the coma which ends fatally in about $10-20 \%$ of the affected cases, about $20-30 \%$ must count on residual damage to the CNS.

Subacute sclerosing panencephalitis (SSPE) is a very rare late complication that manifests on average 6-8 years after infection. On average, there are 4-11 SSPE cases per 100,000 measles diseases. Children under 5 years old are at significantly higher risk. SSPE begins with mental and intellectual changes, develops a progressive course with neurological disorders and deficits to the loss of cerebral functions and ends always lethal.

In immunosuppressed patients often develop severe organ complications which end fatally in $30 \%$ of cases.

In developed countries, the lethality of measles is between $0.05 \%$ and $0.1 \%$, according to the WHO. In the so-called developing countries lethality in some countries is up to $6 \%$.

Diagnostics: Laboratory diagnostics is essential for the reliable detection of measles disease. Serological methods (ELISA, HHT) for the detection of IGM and IGG antibodies are available. Furthermore, a virus genome detection by PCR from urine or throat swab is possible.

Therapy: There is no specific antiviral therapy, only symptomatic therapy is possible.

Prophylaxis: The most effective preventive measure is the live vaccination against measles. A vaccination is possible from the 10th month of age with the measles-mumps-rubella vaccine. Two vaccinations should be administered at least 4 weeks apart. 


\subsection{Rubella Virus}

It is an enveloped RNA virus of the togavirus family. Man is currently the only known host. The rubella virus is distributed worldwide.

Transmission route: Aerogenic by droplet infection. The virus invades the mucous membrane of the upper respiratory tract and subsequently leads to pronounced viremia with the possibility of diaplacental transmission in pregnancy.

Incubation period: 14-21 days.

Duration of contagiousness: It lasts 1 week prior to the onset of the rash and lasts up to 1 week after the appearance of the rash.

Clinical manifestation: In about 50\% of infections, the disease is asymptomatic in childhood. The disease is characterized by a small speckled maculo- or maculopapular rash which begins on the face and subsequently spreads over the body and extremities and disappears after 1-3 days. Other symptoms may include subfebrile temperatures, headache, lymphadenopathy (often nuchal and retroauricular), mild upper airway catarrh and conjunctivitis. Rarely, complications often occur with increasing age (arthritis, bronchitis, otitis, encephalitis, myocarditis). Thrombocytopenia can cause purpura and haemorrhage.

Rubella infection during pregnancy (Congenital Rubella Syndrome): While postnatal rubella infection is rarely associated with complications, infection of the developing foetus through the mother's placenta causes severe damage. The frequency and severity are dependent on the time of infection in infections.

In the first 8 weeks of pregnancy, in $90 \%$ of the cases of infection during the second trimester, in 20-35\% of cases, damage is observed. Primary infection in the first 4 months of pregnancy may result in spontaneous abortion, premature birth or GREGG's triad with defects in the heart (open ductus arteriosus), eyes (cataract) and ears (inner ear deafness). Other possible consequences include lower birth weight, thrombocytopenic purpura, hepatosplenomegaly, encephalitis, hepatitis, myocarditis or microcephaly. While infection of the foetus in the fourth week of gestation may trigger the full picture of the disease, e.g., infection in the 20th week may lead to isolated deafness.

Diagnostics: Detection of virus specific IgM antibodies, e.g., by ELISA. For prenatal diagnosis, a PCR is also available to detect the virus from chorionic biopsy material or amniotic fluid.

Therapy: A specific causal therapy does not exist.

Prophylaxis: Live vaccination see Measles, in addition, unvaccinated women or women with unclear vaccination status of childbearing age should be vaccinated. It is also recommended to vaccinate unvaccinated staff or people with unclear vaccination status in paediatrics, obstetrics, pregnancy care and community facilities.

\subsection{Rabies Virus}

Rabies is a zoonotic disease and is transmitted by an enveloped RNA virus, family of rhabdoviruses. Rabies is common in much of the world. 
Reservoir: Wild animals (foxes, badgers, martens, deer) and domestic animals (cattle, sheep, goats, horses) as well as dogs and cats. Furthermore, bats are an important reservoir. Dogs and cats play an important role as human exposition animals.

Transmission routes: After the infection of the animal, at the end of the incubation period, virus replication occurs in the CNS, where it spreads to the pathogens, whereby the virus is excreted en masse in the saliva. The transmission to humans usually takes place through inoculation via a bite. However, transmission is also possible via skin injury or direct contact with infectious material (e.g. saliva) with the mucosa.

Incubation period: Usually 3-8 weeks, less often shorter than 9 days; in individual cases up to several years. The time to onset of clinical symptoms depends on the location of the bite site. Nearer CNS entry portals describe shorter incubation times. Duration of contagiousness in foxes, dogs and cats are usually 3-7 days before onset of clinical symptoms, as well as throughout the duration of the disease.

Clinical manifestation: The clinic divides into the following stages in humans:

Prodromal stage presents with characteristic discomfort, e.g., headache, loss of appetite, occasional fever. Furthermore, burning, itching and increased pain sensitivity in the area of the bite wound were reported.

Acute neurological phase and encephalitic form: Cerebral dysfunction, further there is a pronounced hydrophobicity. When swallowing, there are spasms of the pharyngeal muscles causing a considerable fear of drinking and the saliva flows out of the mouth. Even the visual or acoustic perception of water leads to restlessness and cramps that can spread to the entire musculature. Furthermore, there are alternating switches between aggressive and depressive moods.

Paralytic form: Increasing onset of paralysis, especially of the cranial nerves.

Coma: In coma and under the signs of respiratory paralysis, death occurs. Usually it takes 7 days between the onset of the first symptoms and the death of untreated patients.

Diagnosis: Suspicion arises first of all from the onset clinical symptoms and a thorough medical history. The antigen or rabies virus detection in epithelial cells of the cornea, in neck skin biopsies, in serum or in cerebrospinal fluid can be tried, but not infrequently it leads to false negative results. Confirmation of the clinical suspected diagnosis will be successful only post-mortem by immunofluorescence from samples of cerebellum and the brainstem.

Preventive action: Pre-exposure immunization by means of rabies vaccination on days $0,7,21$.

Post-exposure measures: The contaminated wound should be immediately and extensively cleaned with water and soap solution (washing out of the pathogen). Furthermore, active and passive immunization against rabies should be performed by vaccination (on days $0,3,7,14,28$ ) and administration of rabies immunoglobulin. 